\section{Polypoid esophageal and gastric metastases of recurrent hepatocellular carcinoma after liver transplantation}

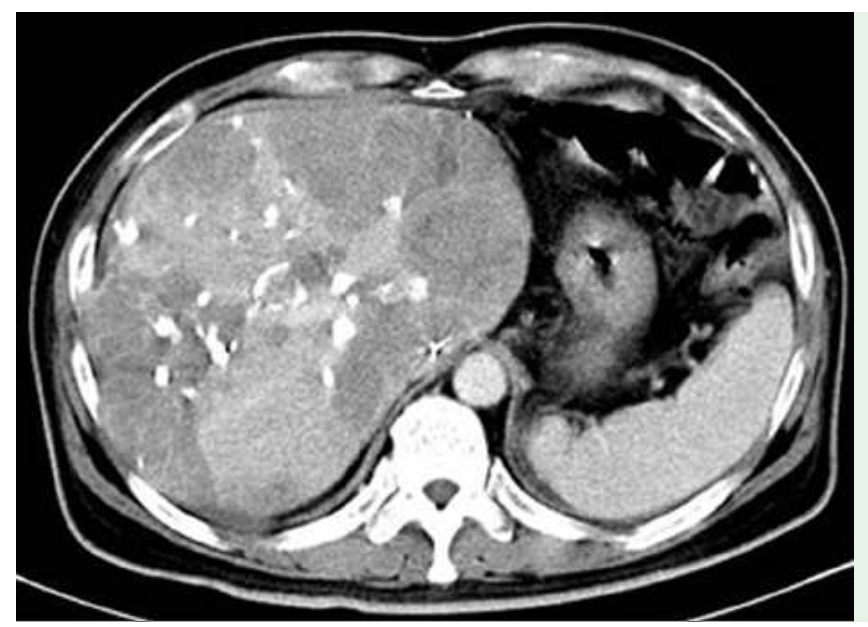

Fig. 1 Abdominal computed tomography (CT) revealed a multinodular mass occupying the right lobe graft and multiple areas of homogeneous hyperdense retention of lipiodol, compatible with the patient's post transarterial chemoembolization (TACE) condition.

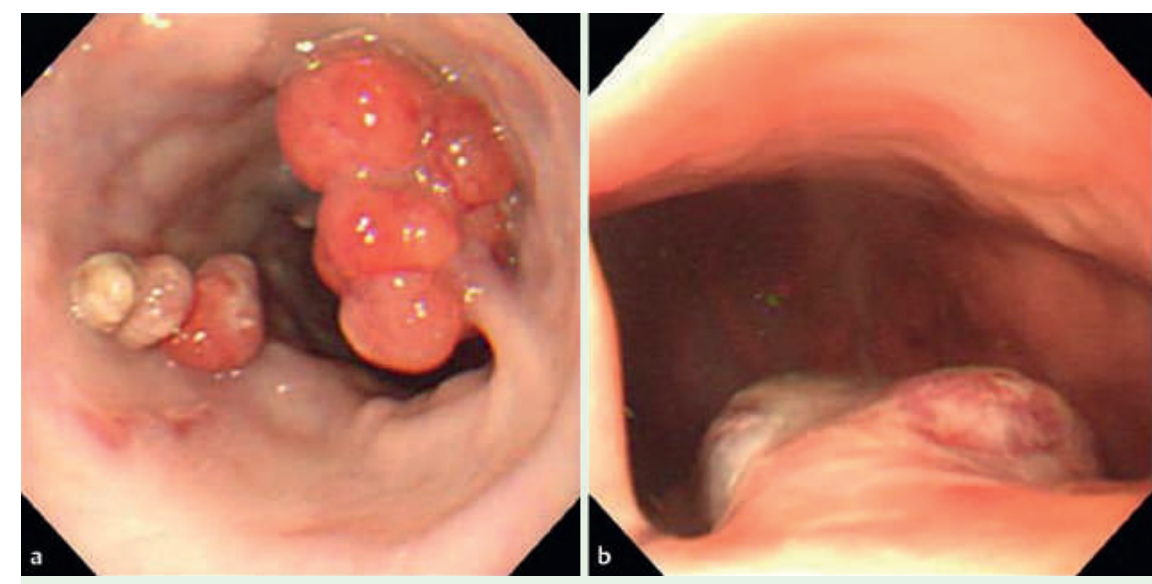

Fig. 2 Endoscopy showed small esophageal varices and some polypoid lesions with congestive mucosa: $\mathbf{a}$ at the middle third of the esophagus; $\mathbf{b}$ at the gastric cardia.
Gastrointestinal tract involvement in hepatocellular carcinoma (HCC) is rare and poor outcomes have been reported [1]. HCC with metastasis to the esophagus is extremely rare and most cases have been diagnosed at postmortem [2]; only a few cases have been visible at endoscopy [35]. We present here a rare case of premortem diagnosis of esophageal and gastric metastases from recurrent HCC, in a liver transplant patient.

A 54-year-old man presented to the emergency department with haematemesis and a 2-day history of tarry stools. He had undergone liver transplantation from a living donor 2 years previously, because of hepatitis B-related cirrhosis and hepatocellular carcinoma. The postoperative course had been uneventful and immunosuppressant drugs such as tacrolimus and mycophenolate mofetil had been prescribed. However, he developed recurrent hepatocellular carcinoma within a year of transplantation and had been treated with thalidomide and transarterial chemoembolization (TACE) (๑ Fig. 1).

On his arrival, physical examination revealed anemic conjunctiva and a distended abdomen. Hemoglobin was $8.1 \mathrm{~g} / \mathrm{dl}$. Panendoscopy showed small esophageal varices, congested-appearing mucosa, and polypoid lesions, located at the middle third of the esophagus and at the gastric cardia ( $\bullet$ Fig. 2). Biopsy of polypoid lesions showed a tumor which was histologically consistent with HCC ( Fig. 3) and which was stained for alphafetoprotein (AFP). The upper gastrointestinal bleeding stopped after conservative treatment. The patient died of disease progression 4 months later.

In the literature, reported cases of esophageal metastasis from HCC presented with major symptoms including gastrointestinal bleeding and dysphagia. The polypoid lesion or submucosal mass is the most common finding at endoscopy. We present here the first reported case of esophageal and gastric metastases of HCC after liver transplantation as a cause of upper gastrointestinal bleeding. The varices seen at endoscopy imply that haematogenous spread is a possible route of metastasis from HCC to esophagus and stomach. Also, the presence of immunosuppressant drugs may facilitate tumor cell metastasis, as in our patient. 


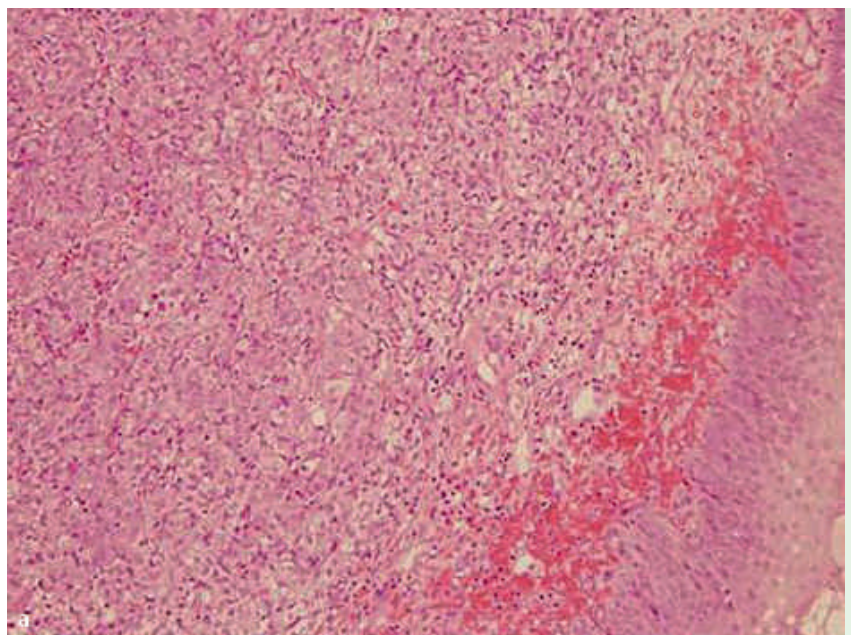

Fig. 3 Photomicrograph of biopsy specimens showing tumor cells with pink cytoplasm, growing in a trabecular and sinusoid pattern (hematoxylin and eosin [H\&E], $\times 200)$ : a esophageal polypoid lesion; $\mathbf{b}$ gastric polypoid lesion.

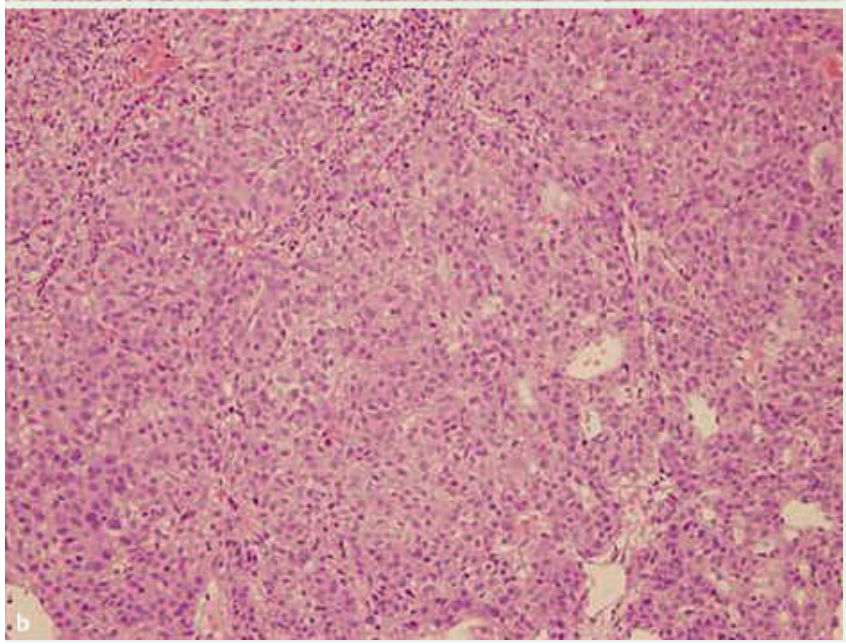

K.-F. Hsu ${ }^{1}$, T.-Y. Hsieh², C.-L. Yeh ${ }^{3}$, M.-L. Shih ${ }^{1}$, C.-B. Hsieh ${ }^{1}$

1 Division of General Surgery, Department of Surgery, Tri-Service General Hospital, National Defense Medical Center, Taipei, Taiwan, Republic of China

2 Division of Hepatogastroenterology, Department of Medicine, Tri-Service General Hospital, National Defense Medical Center, Taipei, Taiwan, Republic of China

3 Department of Surgery, Taoyuan Armed Forces General Hospital, Taoyuan, Taiwan, Republic of China
Bibliography

DOI $10.1055 / \mathrm{s}-0029-1214435$

Endoscopy 2009; 41: E82 -E83

(c) Georg Thieme Verlag KG Stuttgart · New York . ISSN 0013-726X

\section{Corresponding author}

\section{C.-B. Hsieh, MD}

Division of General Surgery,

Department of Surgery

Tri-Service General Hospital

325 Cheng-Kung Rd, Sec 2, Neihu 114

Taipei

Taiwan

Fax: +886-2-87927372

ndmc20470@yahoo.com.tw

\footnotetext{
4 Tsubouchi E, Hirasaki S, Kataoka J et al. Unusual metastasis of hepatocellular carcinoma to the esophagus. Intern Med 2005; 44: 444-447

5 Yan SL, Hung YH, Yang TH. Metastatic hepatocellular carcinoma of the esophagus: an unusual cause of upper gastrointestinal bleeding. Endoscopy 2007; 39 (Suppl 1):

E257-E258

References nal tract involvement in hepatocellular carscopic studies. Endoscopy 1990; 22: 118 123

2 Nakashima T, Okuda K, Kojiro M et al. Patholgy of hepatocellular carcinoma in Japa years). Cancer 1983; 51: $863-877$

3 Kume K, Murata I, Yoshikawa I et al. Polypoid metastatic hepatocellular carcinoma of the esophagus occurring after endoscopic variceal band ligation. Endoscopy 2000; 32: $419-421$
} 